\title{
Optimizing the EBSD signal
}

\author{
Andrew Deal and Alwyn Eades
}

Department of Materials Science and Engineering, Lehigh University, 5 East Packer Avenue, Bethlehem, PA 18015

Electron Backscatter Diffraction (EBSD) analytical equipment has become an important accessory of the scanning electron microscope. The EBSD technique's unique ability to determine rapidly the crystallography of a polycrystalline sample at a submicron level makes it suitable for texture analysis, grain boundary mapping, and phase identification [1]. A limiting factor of EBSD, however, is the signal intensity, which determines the dwell time per point necessary for accurate indexing. To obtain a sufficient signal, the sample is highly tilted as shown in figure 1. Typically, the sample and detector screen are arranged so that $\theta=70^{\circ}, \alpha=0^{\circ}$, and the magnitude of the pattern center vector (PCV) is around $30 \mathrm{~mm}$. Here we use a single scattering Monte Carlo model [2] to simulate the distribution of intensity across the EBSD pattern (diffraction effects are not included) and explore different geometries and sample materials.

A simulated EBSD signal from $\mathrm{Au}\left(20 \mathrm{keV}\right.$ beam, $\left.\theta=70^{\circ}, \alpha=0^{\circ}\right)$ collected in the plane of the detector screen is shown in figure 2. The vertical and horizontal axes of the screen, $\tan (\psi)$ and $\tan (\omega)$, are independent of the PCV length, and the pattern center is identically the origin. The working distance (WD) of the sample, conventionally defined as the distance between the sample and the SEM pole piece along the beam axis, determines the displacement of the screen with respect to the pattern center in this representation. To optimize the EBSD signal for a given experimental configuration, a WD is chosen so that the detector screen, outlined by the circle in figure 2 , is centered on the intensity maximum, as shown. Our simulations demonstrate how the position of this signal maximum for a fixed EBSD geometry can change with material. Figure 3 shows a simulated signal for $\mathrm{Al}$ under the same conditions as for $\mathrm{Au}$ in figure 2. Note that the signal maximum is significantly lower for Al. Specifically, for $|\mathrm{PCV}|=30 \mathrm{~mm}$, the WD must be $\sim 7.5 \mathrm{~mm}$ greater than for $\mathrm{Au}$ to center the screen on the signal maximum. This is because electrons of a given energy will scatter at smaller angles from Al. Table 1 lists this result as well as those for $\mathrm{Cu}$ and $\mathrm{Ag}$, and the change in the optimum WD from the position for Au is given in the last column. Generally, as the atomic number decreases the signal maximum and the pattern center separate.

Our simulations show how an alternative geometry is more advantageous. Figures 4 and 5 show simulated EBSD signals for $\mathrm{Au}$ and Al, respectively, for the geometry: $\theta=60^{\circ}, \alpha=20^{\circ}$. Note that there is a slight increase in the signal collected for both materials, compared with the standard geometry. Additionally, the position of the maximum intensity differs by a smaller distance between materials of different atomic number. The change in the optimum value of the WD between Au and $\mathrm{Al}$ is reduced from $7.5 \mathrm{~mm}$ to $\sim 6.4 \mathrm{~mm}$. Experimentally, the backscatter yield from tilted specimens is known to be highly asymmetric [3]. By tilting the detector screen (changing $\alpha$ ) it is possible to collect more of the electrons onto the screen. The WD adjustment is reduced because the ten-degree difference in sample tilt $(\theta)$ between the two geometries lessens the asymmetry of the backscatter yield. Thus, a geometry of $\theta=60^{\circ}, \alpha=20^{\circ}$ optimizes the EBSD signal collection for a large range of materials with different atomic numbers [4].

\section{References}

[1] A.J. Schwartz et al., Electron Backscatter Diffraction in Materials Science, Plenum Press, New York, 2000.

[2] D.C. Joy, Monte Carlo Modeling for Electron Microscopy and Microanalysis, Oxford University Press, New York, 1995. 
[3] D. Berger and H.Niedrig, Scanning 21 (1999) 187.

[4] Support from DOE, under grant DE-FG02-00ER45819, is gratefully acknowledged.

TABLE 1. Signal maximum.

\begin{tabular}{llll}
\hline \multicolumn{3}{c}{ Atomic } \\
Material & Number & $\psi^{\dagger}$ & $\Delta \mathrm{WD}^{\dagger}$ \\
\hline $\mathrm{Al}$ & 13 & $-31^{\circ}$ & $+7.5 \mathrm{~mm}$ \\
$\mathrm{Cu}$ & 29 & $-27^{\circ}$ & $+4.5 \mathrm{~mm}$ \\
$\mathrm{Ag}$ & 47 & $-24^{\circ}$ & $+3 \mathrm{~mm}$ \\
$\mathrm{Au}$ & 79 & $-19^{\circ}$ & $+0 \mathrm{~mm}$ \\
\hline † For $\theta=70^{\circ}, \alpha=0^{\circ}$, and PCV $=30 \mathrm{~mm}$
\end{tabular}

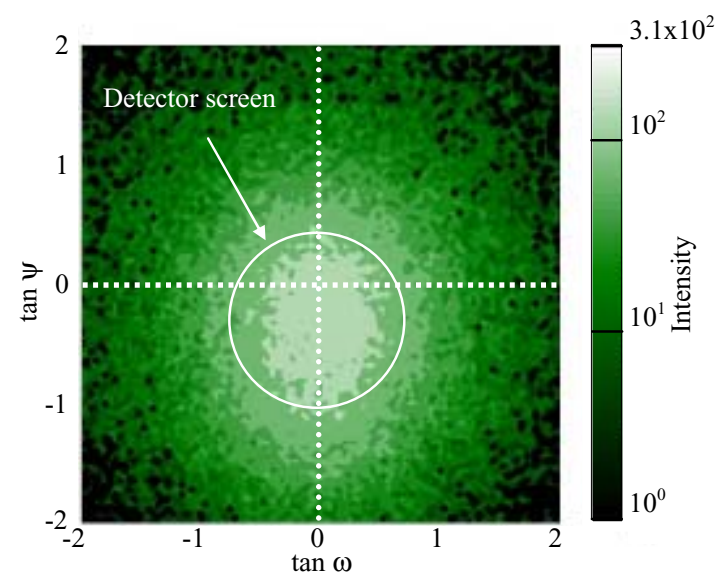

FIG. 2. Simulated Au EBSD signal: $20 \mathrm{keV}$ beam, $\theta=70^{\circ}, \alpha=0^{\circ}$.

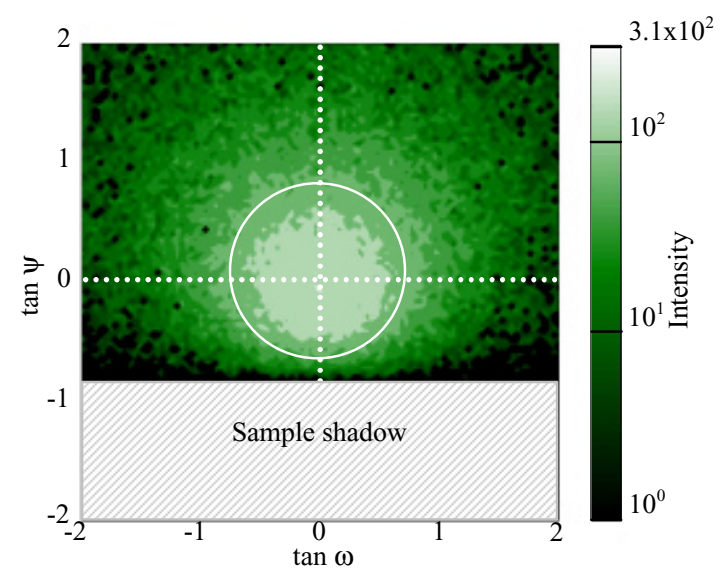

FIG. 4. Simulated Au EBSD signal: $20 \mathrm{keV}$ beam, $\theta=60^{\circ}, \alpha=20^{\circ}$.

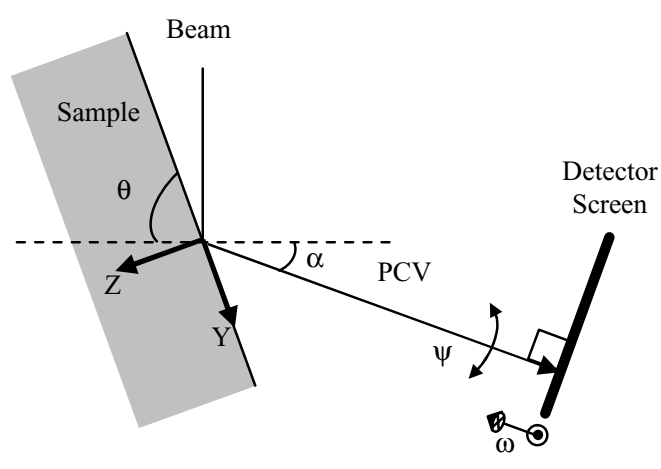

FIG. 1. EBSD Geometry

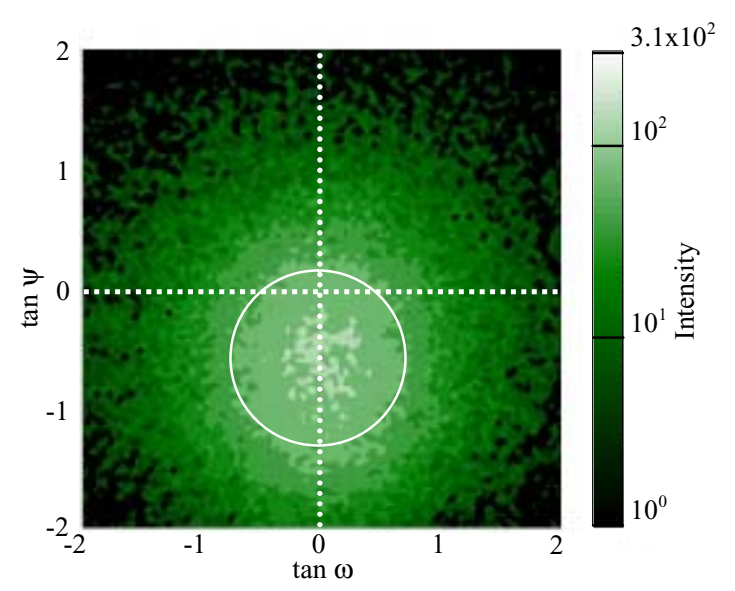

FIG. 3. Simulated Al EBSD signal: $20 \mathrm{keV}$ beam, $\theta=70^{\circ}, \alpha=0^{\circ}$.

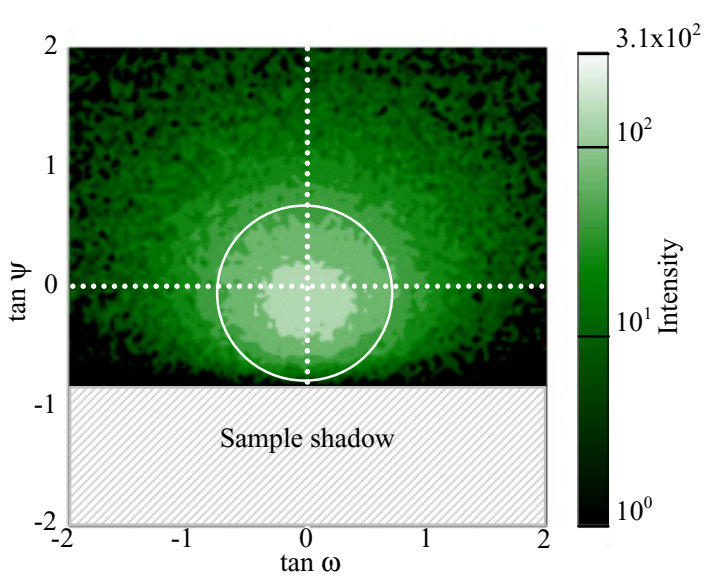

FIG. 5. Simulated Al EBSD signal: $20 \mathrm{keV}$ beam, $\theta=60^{\circ}, \alpha=20^{\circ}$. 